Résumés des conférences et travaux

\title{
Histoire et archéologie des provinces frontières de l'Empire romain
}

Conférences de l'année 2011-2012

\section{Michel Reddé}

\section{(2) OpenEdition Journals}

Édition électronique

URL : https://journals.openedition.org/ashp/1473

DOI : 10.4000/ashp.1473

ISSN : 1969-6310

Éditeur

Publications de l'École Pratique des Hautes Études

Édition imprimée

Date de publication : 1 septembre 2013

Pagination : 82-84

ISSN : 0766-0677

Référence électronique

Michel Reddé, "Histoire et archéologie des provinces frontières de l'Empire romain », Annuaire de I'École pratique des hautes études (EPHE), Section des sciences historiques et philologiques [En ligne], 144 | 2013, mis en ligne le 23 octobre 2014, consulté le 05 novembre 2021. URL : http:// journals.openedition.org/ashp/1473; DOI : https://doi.org/10.4000/ashp.1473 


\title{
HISTOIRE ET ARCHÉOLOGIE DES PROVINCES FRONTIËRES DE L'EMPIRE ROMAIN
}

\author{
Directeur d'études : M. Michel REDDÉ
}

Programme de l'année 2011-2012: Aspects de la romanisation de la Gaule, de la Tène finale à la fin du Ier siècle après J.-C. : le monde rural.

L'année académique 2011-2012 a vu l'inauguration d'un nouveau cycle de conférences consacrées aux campagnes de la Gaule romaine et à leur évolution depuis la fin de l'âge du fer. Il devrait s'étaler sur au moins deux années successives.

On a tout d'abord cherché à poser comme principe fondamental d'une recherche moderne la nécessité d'analyser des systèmes d'exploitation, ce qui suppose de s'appuyer sur des études multiples associant sciences naturalistes et humaines : à cet effet, et grâce à la collaboration de spécialistes invités (pédologie, carpologie, faune), on a mis en évidence l'interactivité entre les pratiques de culture et d'élevage, qui sont ellesmêmes dépendantes des potentialités des sols. Cette approche «agronomique », qui devrait être une évidence, ne l'a pas été jusqu'à présent dans le domaine des sciences de l'Antiquité, et elle sera reprise et développée ultérieurement dans le cadre de ce cycle d'études. Elle a été en revanche abordée très précisément dans le cadre du cours donné par P. Ouzoulias, chargé de recherches au CNRS, et associé à la conférence.

L'historiographie des études sur les campagnes de Gaule montre en effet que les questions posées ont presque toujours, jusqu'à une époque récente, été subordonnées au présupposé d'une ubiquité de la « villa », seule forme reconnue d'exploitation agricole à l'époque romaine. Identifiée aux grands domaines latifundiaires et liée à l'idée d'une appropriation du sol provincial par de nouveaux arrivants « romains » ou par des indigènes fortement romanisés, la «villa » a donc formé le socle des recherches, d'autant plus facilement qu'on pouvait s'appuyer sur les textes des agronomes latins. À cet égard, l'analyse que faisait A. Grenier du fundus de Chiragan, en 1934, est restée un cas d'école (A. Grenier, Manuel d'archéologie gallo-romaine, Paris, 1934, II, 2, p. 859-933). C'est donc la taille du domaine, l'organisation et le luxe des quartiers réservés au dominus et considérés comme une réplique rurale d'un style de vie « urbain » qui ont dominé les études, beaucoup plus que l'analyse de productions pour lesquelles on devait, jusqu'à une époque toute récente, se fonder uniquement sur les textes antiques, pourtant généraux et peu adaptés à l'étude locale des campagnes.

La mise en évidence grâce à la photographie aérienne et la publication, par $\mathrm{R}$. Agache (R. Agache, La Somme pré-romaine et romaine, Amiens, 1978) des villae de la Somme ont constitué une véritable révolution en donnant à voir des paysages antiques que l'on ne soupçonnait guère aussi densément occupés, mais ces découvertes exceptionnelles, bientôt étendues à d'autres régions du Nord de la Gaule, n'ont pas fondamentalement bouleversé la problématique historique des campagnes romaines, 
comme en témoignent les analyses de R. Agache dans les $A N R W$ II, 4, p. 658-713, et celles, contemporaines, de M. Le Glay («La Gaule romanisée », dans G. Duby et A. Wallon [dir.], Histoire de la France rurale, Paris, 1975, p. 191-285). À l'époque, seule E. M. Wightman faisait entendre une voix différente en proposant une analyse plus complexe et un modèle plus nuancé de l'organisation rurale ( $A N R W$, II, 4, p. 584-657).

La recherche sur les cadastres antiques a pris à ce moment-là son plein développement, dominant largement pendant près de deux décennies les études agraires de l'Antiquité. On en a étudié, pendant plusieurs conférences, les principes directeurs, à la lumière des principaux manuels publiés (G. Chouquer, F. Favory, Contribution à la recherche des cadastres antiques, Paris, 1979 [ALUB, 236]; O. A. W. Dilke, The Roman Land Surveyors, New York, 1971, trad. fr. 1995 ; G. Chouquer, F. Favory, Les arpenteurs romains, théorie et pratique, Paris, 1992; G. Chouquer, F. Favory, L'arpentage romain : histoire des textes, droit, techniques, Paris, 2001) et, bien sûr, des documents de la colonie d'Orange (A. Piganiol, Les documents cadastraux de la colonie romaine d'Orange, Paris, 1962 [XVI' suppl. à Gallia]), avant de voir si, en Gaule du Nord, l'ubiquité autrefois postulée du modèle pouvait être reconnue, ce qui n'est pas le cas. Il ne semble donc pas qu'il y ait eu, dans ces régions, appropriation massive des terres pérégrines par des exploitants romains, avec expulsion des indigènes et limitation géométrique des terres provinciales, sauf peut-être dans quelques cas précis, au demeurant difficiles à mettre en évidence aujourd'hui, même dans le cas des colonies.

Dans un deuxième temps, correspondant à peu près au second semestre, la conférence s'est donnée comme objectif d'analyser de manière plus précise, sous un angle très archéologique, des études de villae récemment publiées. On s'est d'abord, dans cette perspective, interrogé sur l'origine protohistorique des grandes exploitations, postulée depuis les années 1990 à l'occasion de différentes fouilles menées en Picardie (D. Bayard, J.-L. Collart [éd.], De la ferme indigène à la villa romaine. La romanisation des campagnes de Gaule. Colloque de Saint-Riquier, 1996). St. Fichtl, professeur à l'université de Tours, est venu à ce propos présenter les recherches de terrain, encore inédites, qu'il mène sur le domaine de la villa de Batilly-en-Gâtinais, daté de la Tène finale, et qui constitue le premier antécédent bien attesté chronologiquement d'une « villa à pavillons multiples », suivant la terminologie actuellement adoptée. Des propositions analogues de développement à partir de structures de la fin de l'âge du fer ont été développées, en Allemagne, pour expliquer la fonction des Viereckschanzen, bien que, dans cette région, la coupure chronologique entre LTD1 et la période romaine soit évidente. On s'est ensuite attaché à examiner les différentes études disponibles, dont la plus grande partie est écrite en allemand, en s'appuyant d'abord sur la typologie récente de K. H. Lenz, qui passe en revue, en les actualisant, les grandes publications classiques sur les villae de Mayen, de Müngersdorf, de Dambach, de Blankenheim, etc., dans lesquelles c'est surtout la typologie de la pars urbana qui est étudiée ("Villae rusticae: zur Entstehung dieser Siedlungsform in den Nordwestprovinzen des römischen Reiches », Köln. Jahrb. 31 [1998], p. 49-70). Bien qu'elle soit au fond assez limitée, une telle approche, fondamentalement morphologique, a le mérite de montrer que le type gallo-romain classique dit « à pavillons d'angle » (Eckrisaliten) n'est 
au fond que le fruit d'une lente évolution d'une forme architecturale simple et plus ramassée, qu'on observe aussi bien en Picardie qu'en Lorraine ou dans le Baden-Württemberg. La publication récente, exhaustive et détaillée des grandes villae à pavillons multiples de Biberist ou de Dietikon, en Suisse, montre en revanche la difficulté qu'on rencontre quand on veut analyser de manière précise la fonction exacte des bâtiments à fonction économique de ces grands ensembles, et sur quels présupposés intellectuels et historiographiques repose leur interprétation. Enfin, dans l'arrière-pays de la CCAA (Cologne), les fouilles menées dans les années 1980 au sein du bassin de lignite ont mis en évidence un réseau dense d'exploitations de taille moyenne, construites avec des matériaux périssables et selon des critères morphologiques assez différents, avec des bâtiments beaucoup plus dispersés dans une cour qui dépasse rarement un hectare. Le paradoxe réside dans le fait que ces établissements ont de bonnes chances, pour une fois, d'être le produit d'un véritable allotissement colonial postérieur à Claude.

Enfin la toute récente publication de N. Roymans et T. Derks (N. Roymans, T. Derks [éd.], Villa Landscapes in the Roman North. Economy, Culture and Lifestyles, Amsterdam, 2011) a fourni l'occasion d'appréhender une autre approche, plus ethno-sociologique, du développement des villae dans le Nord de la Gaule. Poursuivant un fil directeur déjà ancien, mais s'appuyant sur des fouilles récentes et/ou en cours, notamment celle de Tiel-Passewaaij (M. Groot, Animals in ritual and economy in a Frontier community. Excavations in Tiel-Passewaaij, Amsterdam, 2008) les éditeurs de ce colloque montrent, de manière tout à fait novatrice, le fruit que l'on peut tirer d'études qui prennent en compte à la fois les conditions pédologiques des différents ensembles géomorphologiques de cette vaste région, leurs traditions protohistoriques et leur évolution à l'époque romaine pour étudier, dans un cadre large, la «romanisation » de leurs sociétés. 